\title{
Analysis of peri-islet CD45-positive leucocytic infiltrates in long-standing type 1 diabetic patients: additional data regarding cause of death
}

\author{
Shiva Reddy ${ }^{1} \cdot$ Nina Zeng $^{1} \cdot$ Hussam Al-Diery ${ }^{1}$. \\ Doran Jung $^{1} \cdot$ Clifton Yeu $^{1} \cdot$ Maximilian O. Joret ${ }^{1}$ • \\ Mervyn J. Merrilees ${ }^{2}$ - Fiona $\mathrm{Wu}^{3}$
}

Received: 19 May 2015 / Accepted: 21 May 2015 / Published online: 31 May 2015

(C) Springer-Verlag Berlin Heidelberg 2015

To the Editor: In the last column of Table 1 of our recent publication in Diabetologia [1], we stated cause of death as 'not available' for a number of cases we studied. We wish to advise that this missing information can be accessed through the Network for Pancreatic Organ Donors with Diabetes (nPOD) website. Referring to the details on the website, the cause of death for the cases in Table 1 is herewith updated:

Anoxia: Cases 6178, 6134, 6070, 6046, 6089 (with acute pancreatitis), 6049 (with moderate pancreatitis and diabetic ketoacidosis), 6171 (with very mild pancreatitis)

Head trauma: Cases 6123, 6167, 6162, 6113 (changed from 'anoxia'), 6045, 6039, 6197 (with mild multifocal chronic pancreatitis), 6158 (mild focal chronic pancreatitis), 6087 (without secondary complications or pancreatitis)
Cerebral oedema: Case 6052 (diabetic ketoacidosis)

Cerebrovascular/stroke: Case 6172

Duality of interest The authors declare that there is no duality of interest associated with this manuscript.

\section{Reference}

1. Reddy S, Zeng N, Al-Diery H et al (2015) Analysis of peri-islet CD45-positive leucocytic infiltrates in long-standing type 1 diabetic patients. Diabetologia 58:1024-1035

Shiva Reddy

s.reddy@auckland.ac.nz

1 Department of Molecular Medicine and Pathology, Faculty of Medical and Health Sciences, University of Auckland, Private Bag 92019, Auckland, New Zealand

2 Department of Anatomy with Radiology, Faculty of Medical and Health Sciences, University of Auckland, Auckland, New Zealand

3 Diabetes Unit, Auckland District Health Board, Auckland, New Zealand 